Ruben Piepgras*, Sebastian Michlmayr, Johannes Egger und Bernhard G. Zagar

\title{
Potential und Einschränkungen der Messung magnetischer Mikrostrukturen mit einem Faraday-Magnetometer
}

\author{
Potential and limitations of measuring magnetic microstructures with a Faraday rotation \\ magnetometer
}

https://doi.org/10.1515/teme-2019-0122

Eingang 4. September 2019; angenommen 10. September 2019

Zusammenfassung: Magnetische Mikrostrukturen können verwendet werden, um Information zu codieren. Diese Information kann beispielsweise mittels eines FaradayMagnetometers (engl. Faraday Rotation Magnetometer, FRM) zerstörungsfrei analysiert werden. Ein FRM ist ein magneto-optischer Aufbau auf Basis des Faraday-Effekts, der magnetische Feldstärken mit der Drehung polarisierten Lichts verknüpft. In diesem Beitrag wird zunächst ein geschwindigkeitsoptimiertes FRM vorgestellt, das die Magnetisierungen von Strukturen von etwa $60 \mu \mathrm{m}$ Größe qualitativ darstellen kann, jedoch für quantitative Messungen nicht gut geeignet ist. Um das Potential und die Limitierungen eines FRMs als quantitatives Messprinzip zu untersuchen, wird anschließend ein generalisierter Aufbau konzipiert und charakterisiert. Hierfür wird die Amplitudenauflösung und damit die Eignung als quantitatives Messsystem in Abhängigkeit der räumlichen und zeitlichen Auflösung bestimmt. Es werden exemplarisch Methoden der Signalverarbeitung zur Verbesserung der Amplitudenauflösung vorgestellt und ausgewertet. Des Weiteren wird gezeigt, wie sich daraus negative Auswirkungen auf die räumliche beziehungsweise zeitliche Auflösung ergeben können. Aus diesen Zusammenhängen lässt sich zunächst für eine gegebene Problemstellung bezüglich geforderter räumlicher, zeitlicher und quantitativer Auflösung abschätzen, ob ein FRM ein geeignetes Messsystem darstellt. In der Folge helfen diese Überlegungen außerdem beim Aufbau sowie der Wahl der Komponenten des Magnetometers.

*Korrespondenzautor: Ruben Piepgras, Johannes Kepler Universität Linz, Institut für Elektrische Messtechnik, Altenberger Straße 69, 4040 Linz, Austria, E-Mail: ruben.piepgras@jku.at

Sebastian Michlmayr, Johannes Egger, Bernhard G. Zagar, Johannes Kepler Universität Linz, Institut für Elektrische Messtechnik, Altenberger Straße 69, 4040 Linz, Austria
Schlagwörter: Magneto-optische Verfahren, FaradayRotation, magnetische Mikrostrukturen, zerstörungsfreie Prüfung.

Abstract: Magnetic microstructures can be used to encode information. This information can then be analyzed nondestructively using, e.g. a Faraday Rotation Magnetometer (FRM). An FRM is a magneto-optical setup built around the Faraday effect, which links magnetic field strengths with the rotation of polarized light. In this contribution we first present an acquisition-speed-focused FRM. With the help of this setup it is possible to qualitatively resolve structures of about $60 \mu \mathrm{m}$ in size. However, it does not allow any meaningful quantitative measurements. Therefore, we built and characterized a generalized setup in order to establish the potential and limitations of an FRM as a quantitative measurement device. We determine the interdependence of amplitude, spatial, and temporal resolution. Afterwards, we present ways to enhance the signal w. r.t. amplitude resolution, i.e. the suitability for quantitative measurements, as well as their detrimental effects on spatial and temporal resolution. Given a set of requirements, this allows for an estimate of feasibility for an FRM as a quantitative measurement device. Furthermore, it helps in the build as well as the selection of components of the magnetometer.

Keywords: Magneto-optics, Faraday rotation, magnetic microstructures, non-destructive testing.

\section{Einleitung}

Das Codieren von Information mittels magnetischer Mikrostrukturen findet unter anderem in Magnetstreifenkarten und Banknoten Anwendung. So verlangen beispielsweise viele Zentralbanken - darunter die Europäische Zentralbank - den Einsatz sogenannter Sicherheitsfäden, um die Fälschungssicherheit und Maschinenlesbarkeit der Banknoten zu verbessern. Der Sicherheitsfa- 
den ist ein wenige Millimeter breiter Streifen, der mit dem Papier der Banknote verwoben ist. Dieser Streifen besteht aus einer Polyethylen-Folie, auf die unter anderem gemusterte Magnetstreifen sowie Maskierungsschichten gedruckt werden. Die Maskierungsschichten dienen dazu, eine rein optische Inspektion der Muster auf den Magnetstreifen zu verhindern. Die Muster dieser Streifen haben bei Eurobanknoten aktuell eine Strukturgröße von etwa $60 \mu \mathrm{m}$ und bestehen aus zwei unterschiedlichen Materialien, die sich in ihrer Remanenz um etwa eine Größenordnung unterscheiden. Um die Magnetstreifen im Umlauf befindlicher Banknoten zu prüfen oder deren Qualität in der Herstellung zu sichern, müssen sie zunächst magnetisiert und anschließend vermessen werden. Das dafür verwendete Messsystem muss insbesondere für schnelle Prüfungen zerstörungs- und abschabungsfrei arbeiten. In [1], [2] und [3] werden verschiedene magneto-resistive und -optische Sensoren vorgestellt, die potentiell verwendet werden könnten, aber für die Aufgabenstellung optimiert werden müssten. Ausgehend von [3] wird in diesem Beitrag ein Faraday-Magnetometer (engl. Faraday Rotation Magnetometer, FRM) näher betrachtet. Ein FRM ist ein magneto-optischer Aufbau auf Basis des FaradayEffekts. Der Faraday-Effekt beschreibt, wie sich die Polarisationsebene linear polarisierten Lichts beim Durchgang durch gewisse Medien (Medien, bei denen dieser Effekt besonders stark auftritt, werden Faraday-Kristalle genannt) dreht, sofern diese einem Magnetfeld in Richtung der Lichtausbreitung ausgesetzt sind. Durch die Bestimmung des Rotationswinkels können bei Kenntnis der Kristalleigenschaften Rückschlüsse auf externe Magnetfelder gezogen werden. Fasst man nun die Magnetisierungen der $\mathrm{zu}$ untersuchenden Struktur als Ausgangspunkte dieser Magnetfelder auf, erlaubt ein FRM somit die Untersuchung der magnetischen Mikrostrukturen.

Im Folgenden wird zunächst die einem FRM zugrundeliegende Theorie erläutert. Anschließend wird ein Aufbau vorgestellt, der speziell für die Qualitätssicherung in der Produktion der Sicherheitsfäden ausgelegt wurde. Dieser ist für die schnelle Erfassung der beiden in ihrer Magnetisierung stark unterschiedlichen Materialien im Muster ausgelegt. Der Aufbau erfüllt diese Anforderungen, ist darüber hinaus aber nicht für quantitative Messungen geeignet. Daher wird im Anschluss ein generalisierter Aufbau präsentiert und charakterisiert. Anhand dieses Aufbaus werden möglichst allgemein das Potential und die Limitierungen des FRMs als quantitatives Messsystem bestimmt. Dabei werden verschiedene Methoden zur Signalbearbeitung aufgezeigt und deren Auswirkung auf Zeit-, Orts- und Amplitudenauflösung sowie deren gegenseitige Abhängigkeit analysiert. Die Charakterisierung des Auf- baus und der nachfolgenden Signalverarbeitungsschritte soll dazu dienen, abschätzen zu können, ob ein FRM bezüglich gegebener Anforderungen für quantitative Messungen geeignet ist. Des Weiteren soll dies im Falle einer Eignung bei der Auswahl der Komponenten behilflich sein.

\section{Grundlegender Aufbau und theoretischer Hintergrund}

Der prinzipielle Aufbau eines FRMs ist in Abbildung 1 dargestellt. Ein FRM ist um einen sogenannten FaradayKristall herum aufgebaut. Ein Faraday-Kristall ist ein Medium, bei dem die magneto-induzierte zirkulare Doppelbrechung - genannt Faraday-Effekt - besonders ausgeprägt ist. Der Faraday-Effekt beschreibt hierbei die Rotation der Polarisationsebene linear polarisierten Lichts innerhalb des Kristalls, wenn dieser einem externen magnetischen Feld in Lichtausbreitungsrichtung ausgesetzt ist.

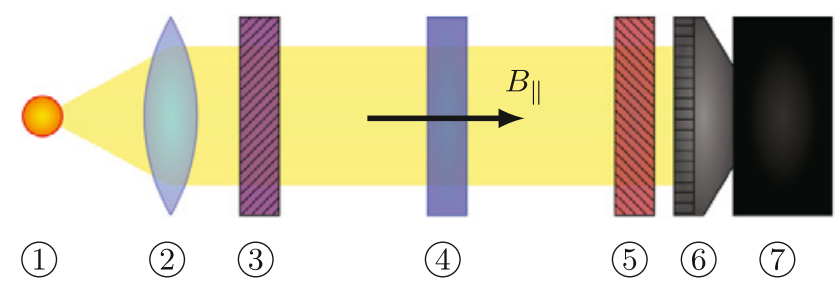

Abb. 1: Prinzipieller Aufbau eines FRMs: (1) Lichtquelle, (2) Kollimator, (3) Polarisator, (4) Faraday-Kristall, (5) Analysator, (6) Objektiv, (7) Kamera.

Für eine magnetische Flussdichtenkomponente $B_{\|}$in Ausbreitungsrichtung des Lichts und eine Kristalldicke $d$ gilt für den Drehwinkel

$$
\beta=B_{\|} \cdot d \cdot V,
$$

wobei $V$ als Verdet-Konstante bezeichnet wird und materialspezifisch ist [4]. Bei der Vermessung nichttransparenter Schichten muss das FRM so konzipiert werden, dass es von einer frei zugänglichen Seite aus verwendet werden kann. Hierbei wird eine Seite des Kristalls verspiegelt und das Licht durchläuft diesen zweimal. Aufgrund von Doppelbrechung [5] verdoppelt sich die Drehung zwischen Ein- und Austritt des Lichts $\mathrm{zu} \beta^{\dagger}=2 \beta$. Von einer solchen Anordnung wird in der Folge ausgegangen. Bei Kenntnis der Kristalleigenschaften $d$ und $V$ 
sowie der Messung des Rotationswinkels $\beta^{\dagger}$ ließe sich die magnetische Flussdichte über

$$
B_{\|}=\frac{\beta^{\dagger}}{2 \cdot d \cdot V}
$$

bestimmen. Allerdings lässt sich der Rotationswinkel nicht direkt messen. Stattdessen werden im FRM zwei Polarisationsfilter eingesetzt - ein Polarisator und ein Analysator. Der Polarisator erzeugt aus unpolarisiertem Licht linear polarisiertes Licht der Intensität $I_{0}$. Das polarisierte Licht wird im Kristall um $\beta^{\dagger}$ gedreht und durchläuft den Analysator, der im Winkel $\alpha$ zum Polarisator steht. Die Lichtintensität $I_{\alpha, \beta^{\dagger}}$ nach dem Analysator lässt sich nach dem Gesetz von Malus über

$$
I_{\alpha, \beta^{\dagger}}=I_{0} \cdot \cos ^{2}\left(\alpha+\beta^{\dagger}\right)=I_{\alpha} \cdot \frac{\cos ^{2}\left(\alpha+\beta^{\dagger}\right)}{\cos ^{2}(\alpha)}
$$

bestimmen [6]. Hierbei beschreibt $I_{\alpha}$ die Intensität nach dem Analysator, wenn kein externes Magnetfeld anliegt. Mit den Zusammenhängen aus Gleichung 1 beziehungsweise 2 und Gleichung 3 lässt sich somit aus einer Intensitätsmessung die magnetische Flussdichte beziehungsweise Feldstärke bestimmen. Eine zweidimensionale Messung erlaubt schließlich die Bestimmung der relativen räumlichen Magnetisierungsverteilung einer Probe.

\section{Geschwindigkeitsoptimierte qualitative Messungen}

In diesem Abschnitt wird ein Aufbau vorgestellt, der speziell für die Echtzeit-Qualitätssicherung in der Herstellung von Sicherheitsfäden für Banknoten konzipiert wurde und eingesetzt wird [7]. Dieser Aufbau erlaubt das Erkennen von Magnetisierungsstrukturen mit einer Strukturgröße größer gleich $60 \mu \mathrm{m}$ und einem Unterschied in der magnetischen Remanenz von einer Größenordnung. Insbesondere können bis zu 65000 mit 64 Pixeln aufgelöste Messungen pro Sekunde kontinuierlich über einen Zeitraum von mehreren Stunden durchgeführt und lückenlos zur Qualitätssicherung aufgezeichnet werden.

Das Prinzip der hier vorgestellten Qualitätssicherung in der Herstellung der Sicherheitsfäden ist in Abbildung 2 dargestellt. Die Sicherheitsfäden für Eurobanknoten werden in einem Rolle-zu-Rolle-Verfahren produziert. Hierbei wird eine $50 \mu \mathrm{m}$ dicke Polyethylenfolie mit einer Geschwindigkeit von $v=3 \mathrm{~m} \mathrm{~s}^{-1}$ bewegt. Auf diese werden unter anderem die nicht-transparenten magnetischen Strukturen mit einer Dicke von etwa $500 \mathrm{~nm}$ und einer

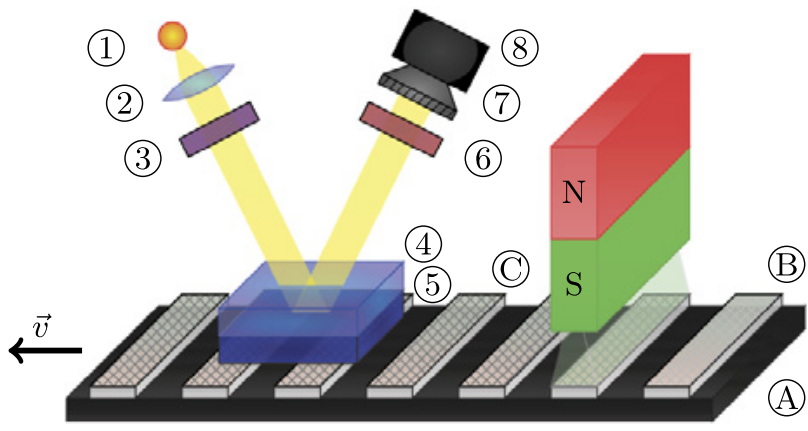

Abb. 2: Prinzip der Qualitätssicherung während der Herstellung (nicht maßstabsgetreu): mit Strukturen bedruckte Polyethylenfolie (A) bewegt sich mit Geschwindigkeit $\vec{v}$; unmagnetisierte Strukturen (B) werden magnetisiert; magnetisierte Strukturen (C) werden mit FRM (1) - 8) vermessen; Bestandteile des FRMs: (1) Lichtquelle, (2) Kollimator, (3) Polarisator, (4) Faraday-Kristall, (5) Verspiegelung, (6) Analysator, (7) Objektiv, (8) Kamera.

Strukturgröße von etwa $60 \mu \mathrm{m}$ gedruckt. Das unterscheidende Merkmal innerhalb der Struktur sind dabei die magnetischen Remanenzen der verwendeten Materialien. Es werden zwei unterschiedliche Materialien mit einer magnetischen Remanenz von etwa $30 \mathrm{mT}$ respektive $300 \mathrm{mT}$ eingesetzt. Für diese Strukturen soll im laufenden Betrieb über mehrere Stunden kontinuierlich eine Qualitätssicherung durchgeführt werden. Hierbei stellt insbesondere die gewünschte Geschwindigkeit hohe Anforderungen an die Komponenten des FRMs sowie an die nachfolgenden Schritte zur Datenverarbeitung und -speicherung.

Der Faraday-Kristall muss aufgrund der NichtTransparenz der Folie verspiegelt sein. Da die von den Magnetisierungen verursachte magnetische Flussdichte örtlich sehr schnell abfällt, muss der Abstand von Folie und Kristall so gering wie möglich gehalten werden. Dafür wiederum muss die Spiegelschicht möglichst dünn sein und die Folie direkt kontaktieren. Aufgrund des Kontaktes muss die Spiegelschicht glatt sein, um die bewegte Folie nicht zu beschädigen. Ebenfalls wegen des schnellen Abfalls der magnetischen Flussdichte sollte der Kristall möglichst dünn sein. Um der daraus resultierenden Verringerung der Faraday-Rotation entgegenzuwirken, muss der Kristall eine sehr hohe Empfindlichkeit aufweisen. Im Aufbau wird ein Kristall der Firma Matesy [8] eingesetzt. Dieser besteht aus einem Substrat, auf dem eine Faradayaktive, eine spiegelnde und eine schützende Schicht aufgebracht sind. Die dielektrische Spiegel- und die Schutzschicht haben insgesamt eine Dicke von $5 \mu \mathrm{m}$ und sorgen damit für einen ausreichend kleinen Abstand der Faradayaktiven Schicht zur Probe. Die Schutzschicht besteht aus chemisch abgelagertem Diamant und erfüllt die Forderung nach geringer Oberflächenrauheit. Die Faraday-aktive 
Schicht besteht aus einem mit Seltenerd-Elementen substituiertem Bismut-Eisen-Granat. Sie hat ebenfalls eine Dicke von $5 \mu \mathrm{m}$ und ist für eine Verwendung im Bereich von $\pm 2 \mathrm{kA} \mathrm{m}^{-1}$ beziehungsweise $\pm 4^{\circ}$ ausgelegt. Ihr hervorstechendstes Merkmal ist ihre extrem hohe Empfindlichkeit von $V \approx 2,5 \cdot 10^{6} \mathrm{rad} \mathrm{T}^{-1} \mathrm{~m}^{-1}$. Im Vergleich weisen typischerweise aufgrund ihrer hohen Empfindlichkeit eingesetzte Materialien wie Terbium-Gallium-Granat eine um vier Größenordnungen geringere Verdet-Konstante auf [9].

Die Bedingungen für Lichtquelle und Kamera können nicht vollständig getrennt voneinander betrachtet werden. Für die Kamera folgt aus der gewünschten Mindestauflösung von $60 \mu \mathrm{m} \times 60 \mu \mathrm{m}$ und der Geschwindigkeit der Folie von $3 \mathrm{~m} \mathrm{~s}^{-1}$ eine untere Schranke für die Bildfrequenz von 50000 Bildern pro Sekunde. Aufgrund der daraus folgenden maximalen Belichtungszeit von $20 \mu$ s müssen einerseits die Empfindlichkeit der Kamera und andererseits die eingehende Lichtintensität möglichst hoch sein. Die Intensität der Szenenbeleuchtung kann allerdings nicht beliebig hoch gewählt werden, da sonst der Kristall thermisch beschädigt werden könnte. Im Aufbau wird die Kombination aus einer Hamamatsu S11106-10 Zeilenkamera und einer OSRAM LRW55N Hochleistungs-LED verwendet. Die Kamera verfügt über 128 Pixel mit einer jeweiligen Größe von $63,5 \mu \mathrm{m} \times 63,5 \mu \mathrm{m}$ und einer Empfindlichkeit von $80 \mathrm{Vlx}^{-1} \mathrm{~s}^{-1}$. Ein Linienscan kann hiermit in etwa $13 \mu \mathrm{s}$ durchgeführt werden. Die Lichtquelle ist eine WeißlichtLED mit einer Peakwellenlänge von $625 \mathrm{~nm}$. Deren Lichtstrom beträgt $192 \mathrm{~lm}$ bei einem Diodenstrom von $1 \mathrm{~A}$. Die Beleuchtungsstärke durch die LED nähert sich den Grenzen der thermischen Belastung des Kristalls, nutzt aber nur etwa $30 \%$ des Messbereichs der Kamera aus. Der damit einhergehende Verlust an Amplitudenauflösung ist für diesen Anwendungsfall noch akzeptabel, da nur verhältnismäßig grobe Unterscheidungen der Intensitäten benötigt werden.

Weitere sehr große Herausforderungen innerhalb des Messablaufs betreffen die dem FRM nachfolgenden Schritte zur Datenbearbeitung, -weitergabe und -speicherung. Hierbei wird im Messaufbau zunächst das Kamerasignal mit einer $10 \mathrm{MHz}$-Taktrate abgerufen. Dieses wird analog verstärkt, mit einem 8-Bit-ADC digitalisiert und in einen FIFO-Buffer geschrieben. Der Buffer wird von einem Raspberry Pi ausgelesen und die Daten werden schließlich auf einer Solid State Disk (SSD) gespeichert. Als äußerst anspruchsvoll erwies sich hier die durch die hohe Scanrate bedingte kontinuierliche Datentransferrate zur SSD von $8 \mathrm{MB} / \mathrm{s}$. Diese musste bei der Speicherung nicht nur als Burstrate, sondern als konstante Datenrate für eine Dauer von mindestens vier Stunden zur Verfügung stehen. Dies konnte im Aufbau umgesetzt werden, allerdings nur bei einer Verringerung der Linienscanbreite auf 64 Pixel.

Mit diesem Aufbau können mehrstündige kontinuierliche Qualitätssicherungen bei der Herstellung von Banknoten-Sicherheitsfäden im laufenden Betrieb durchgeführt werden. Abbildung 3 zeigt einen Ausschnitt eines damit erzielten Messergebnisses. Das Testmuster der Magnetisierungsstruktur ist hier deutlich zu erkennen. Die Amplitudenauflösung für die eingehende Intensität ist ausreichend, um zwischen den unterschiedlichen Magnetisierungen $\mathrm{zu}$ differenzieren. Allerdings unterscheiden sich die Magnetisierungen um eine Größenordnung und die Unterscheidbarkeit der Strukturen ist für kleinere Differenzen nicht mehr gegeben. Somit kann der Aufbau nur für qualitative, nicht aber für sinnvolle quantitative Messungen eingesetzt werden. Hinsichtlich anderer Messprobleme oder auch zukünftiger Entwicklungen des gleichen Messproblems ist es aber dennoch von Interesse, inwieweit ein FRM für quantitative Messungen eingesetzt werden kann.

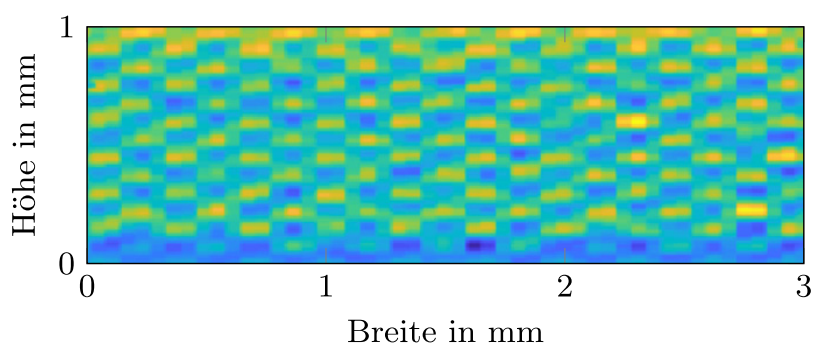

Abb. 3: Mit einem FRM nach [7] während der Herstellung von Banknoten-Sicherheitsfäden erzeugte Aufnahme eines $3 \mathrm{~mm}$ breiten Test-Streifens: Gelb kennzeichnet Bereiche höherer, blau niedrigerer Magnetisierung.

\section{Potential und Einschränkungen bei quantitativen Messungen}

In diesem Abschnitt wird diskutiert, inwiefern ein FRM ein geeigneter Messaufbau für quantitative Messungen ist. Hierfür wird ein verallgemeinerter Aufbau vorgestellt und charakterisiert. Anschließend werden Methoden zur digitalen Signalverarbeitung analysiert. Diese Methoden zielen darauf ab, die Amplitudenauflösung und damit die Eignung des Aufbaus für quantitative Messungen zu verbessern. Da die Verbesserung der Amplitudenauflösung teilweise nur auf Kosten der räumlichen oder zeitlichen Auflösung erreicht werden kann, wird schließlich diese gegenseitige Abhängigkeit quantitativ ausgewertet. 


\subsection{Messaufbau zur Systemcharakterisierung}

Abbildung 4 zeigt schematisch den generalisierten Aufbau zur Charakterisierung des Gesamtsystems. Wie auch der in Abschnitt 3 vorgestellte Aufbau ist er für die Anwendung auf nicht-transparente Medien ausgelegt. Es wird der gleiche Faraday-Kristall mit einer Verdet-Konstante von $V=2,5 \cdot 10^{6} \mathrm{rad} \mathrm{T}^{-1} \mathrm{~m}^{-1}$ und einem angegebenen Arbeitsbereich von $\pm 2 \mathrm{kA} \mathrm{m}^{-1}$ beziehungsweise $\pm 4^{\circ}$ verwendet. Als Lichtquelle wird eine einfarbige Engin LZ1-00A102 Hochleistungs-LED mit einer zur maximalen Empfindlichkeit des Kristalls passenden Wellenlänge von $590 \mathrm{~nm}$ eingesetzt. Im Gegensatz zum vorherigen FRM spielt die Bildwiederholrate bei diesem Aufbau lediglich eine untergeordnete Rolle. Stattdessen soll er anhand zweidimensionaler Messungen weitestgehend statischer Proben charakterisiert werden. Daher wird die Zeilenkamera durch die 2D-Kamera Allied Vision Mako G223B ersetzt. Hierbei handelt es sich um eine Schwarzweiß-Kamera mit einer Auflösung von $2058 \times 1088$ Pixeln und einer Pixelgröße von $5,5 \mu \mathrm{m} \times 5,5 \mu \mathrm{m}$. Die Amplitudenauflösung ist durch den verwendeten Bereich des ADCs von 8 bit festgelegt. Die Bildwiederholrate der Kamera beträgt 49,5 fps. Dies ist vergleichsweise wenig, allerdings können die nachfolgenden

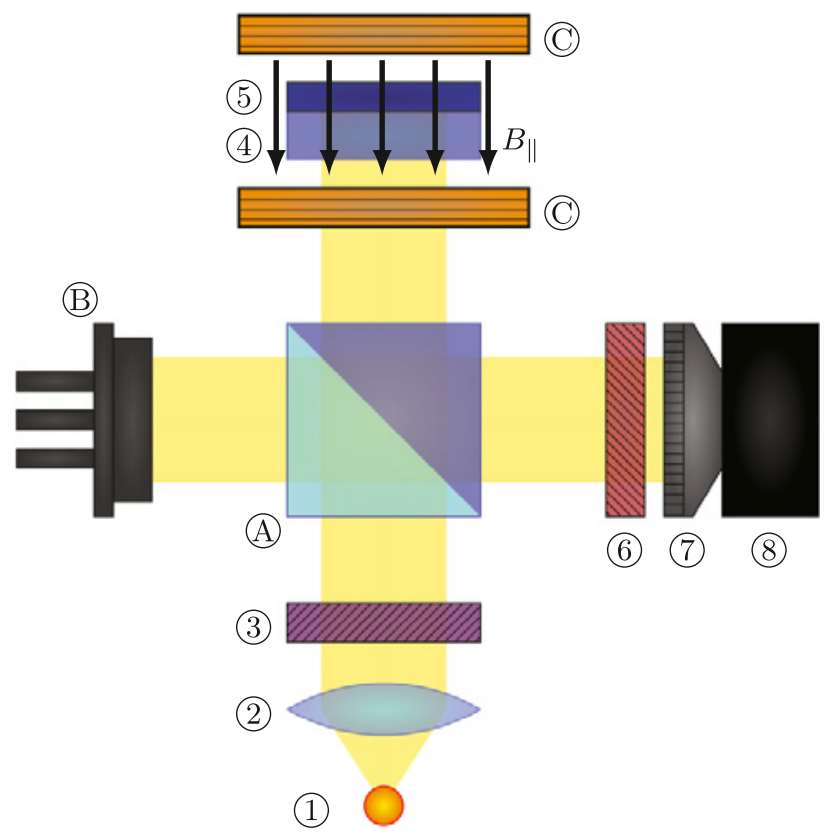

Abb. 4: Schematischer Aufbau des generalisierten FRMs mit (1) Lichtquelle, (2) Kollimator, (3) Polarisator, (4) Faraday-Kristall, (5) Verspiegelung, (6) Analysator, (7) Objektiv und (8) Kamera sowie den Erweiterungen zur Charakterisierung (A) Strahlteiler, (B) Fotodiode und (C) Helmholtzspule.
Überlegungen für die Verbesserung der zeitlichen Auflösung beziehungsweise deren Zusammenspiel mit der Amplitudenauflösung auf beliebige Kameras übertragen werden. Die Polarisationsfilter sind in einem relativen Winkel von $\alpha=45^{\circ}$ zueinander angeordnet. Dies maximiert nach dem Gesetz von Malus in Gleichung 3 die absolute Empfindlichkeit der Intensität bezüglich einer Änderung des Polarisationswinkels $\beta^{\dagger}$. Für einen Arbeitsbereich $\Delta \beta^{\dagger}= \pm 4^{\circ}$ des Polarisationswinkels lässt sich der Arbeitsbereich $\Delta I_{W}$ der einfallenden Intensität an der Kamera bestimmen, wobei

$$
\Delta I_{W} \in\left[I_{\min }, I_{\max }\right] \approx\left[\left(I_{45^{\circ}} \times 0,86\right),\left(I_{45^{\circ}} \times 1,14\right)\right]
$$

gilt. Aufgrund des Offsets $I_{\min }$ nimmt der Arbeitsbereich $\Delta I_{W}$ der einfallenden Intensität nur einen kleinen Teil des Gesamt-Messbereiches $\Delta I_{C}$ der Kamera ein. Unter Berücksichtigung der Amplitudenauflösung des ADCs der Kamera kann daher aufgrund von Quantisierungsrauschen ein einzelnes Bild bestenfalls zu 2,8\% des Arbeitsbereiches $\Delta I_{W}$ der einfallenden Intensität aufgelöst werden.

Das FRM nach Abbildung 4 ist in seiner Konfiguration im Vergleich zum vorherigen Aufbau nach Abbildung 2 weiterhin dahingehend abgewandelt, dass die Möglichkeiten zur Charakterisierung des Systems verbessert sind. So kann hier zum einen ebenfalls die Intensität $I_{0}$ vor dem Faraday-Kristall gemessen und gegebenenfalls stabilisiert werden. $\mathrm{Um} I_{0} z u$ bestimmen, wird mittels eines Strahlteilers der Lichtpfad in einen Referenz- und einen Messpfad aufgeteilt. Die Intensität im Referenzpfad wird mit einer Fotodiode des Typs Burr-Brown OPT301M aufgezeichnet. Das Licht im Messpfad durchläuft nach dem Strahlteiler den Kristall, wird gespiegelt und durchläuft abermals Kristall und Strahlteiler sowie den Analysator. Anschließend wird das vom Kristall erfasste Magnetbild optisch scharfgestellt auf die Kamera abgebildet. Zum anderen kann im Aufbau nach Abbildung 4 nun die magnetische Feldstärke $H_{\|}$beziehungsweise Flussdichte $B_{\|}$am Faraday-Kristall und damit indirekt die Faraday-Rotation $\beta^{\dagger}$ eingestellt werden. Hierfür wird eine Helmholtzspule eingesetzt, die die Rolle der magnetischen Probe übernimmt. Sie ist besonders für die Charakterisierung geeignet, da die magnetische Feldstärke innerhalb von Helmholtzspulen nahe deren Achsen als nahezu homogen betrachtet werden kann und ihr Betrag über den Spulenstrom sehr genau einstellbar ist.

Innerhalb dieses Aufbaus erwies sich das Verhalten der LED, der Kamera sowie des Faraday-Kristalls als besonders kritisch für Amplitudenauflösungen, die sinnvolle quantitative Messungen erlauben würden. Im Folgenden werden das Verhalten der Amplitudenauflösung ge- 
zeigt sowie hard- und softwareseitige Möglichkeiten zu ihrer Verbesserung vorgestellt und ausgewertet. Dabei wird auch aufgezeigt, welche negativen Auswirkungen die Methoden zur Verbesserung der Amplitudenauflösung auf die zeitliche und räumliche Auflösung haben können.

\subsection{Signalrauschen der Kamera}

Das Ausgangssignal der Kamera weist für ein einzelnes Bild sehr starkes Rauschen auf. Dieses nimmt aufgrund der sehr geringen Faraday-Rotation $\beta^{\dagger}$ und deren Abbildung auf die Intensität $I_{\alpha, \beta^{\dagger}}$ über das Gesetz von Malus nach Gleichung 3 bis zu 40\% des Arbeitsbereiches $\Delta I_{W}$ der einfallenden Intensität ein. Allerdings kann die statistische Unabhängigkeit der einzelnen Bilder verwendet werden, um die Amplitudenunschärfe zu verringern. Die Verwendung eines zeitlichen Moving-Average-Filters mit der Filterlänge $N$ beispielsweise verringert die Standardabweichung der Amplitude um einen Faktor $\sqrt{N}$. Andererseits verlängert eine solche Mittelung in den meisten Anwendungsfällen die Aufnahmedauer um den Faktor $N$. In der Folge werden Messergebnisse, sofern nicht anders angegeben, jeweils mit einer Filterlänge von $N=100$ dargestellt.

\subsection{Signaldrift von Kamera und LED}

Zusätzlich zum Rauschen ist das Ausgangssignal der Kamera starkem Drift unterworfen. Dies wurde hauptsächlich auf Temperatureinflüsse auf LED und Kamera zurückgeführt. Da ein unbeachteter Drift im Signal zu verfälschten Ergebnissen in der Berechnung des Rotationswinkels $\beta^{\dagger}$ führen würden, ist es wichtig entweder die Ursachen für den Drift zu unterdrücken oder das Kamerasignal entsprechend rechnerisch zu korrigieren. Für das vorgestellt Messsystem wurde das Kamerasignal korrigiert, anstatt beispielsweise die Temperatur von LED und Kamera mittels Peltier-Elementen zu regeln. Hierfür wurde zum einen das Signal der Fotodiode im Referenzarm und zum anderen der Temperatursensor der Kamera sowie die vom Hersteller angegebene temperaturabhängige Empfindlichkeit verwendet. Abbildung 5 zeigt das Langzeitverhalten der aus dem Ausgangssignal der Kamera berechneten Rotation bezüglich eines einzelnen Pixels. Hierbei wurde ein zeitliches Moving-Average-Filter der Länge $N=1000$ eingesetzt. Es sind das unkorrigierte Signal, das mit dem Signal der Fotodiode sowie das zusätzlich mit dem Kamera-Temperatursensor korrigierte Signal angegeben. Es lag zu keinem Zeitpunkt ein externes Magnetfeld

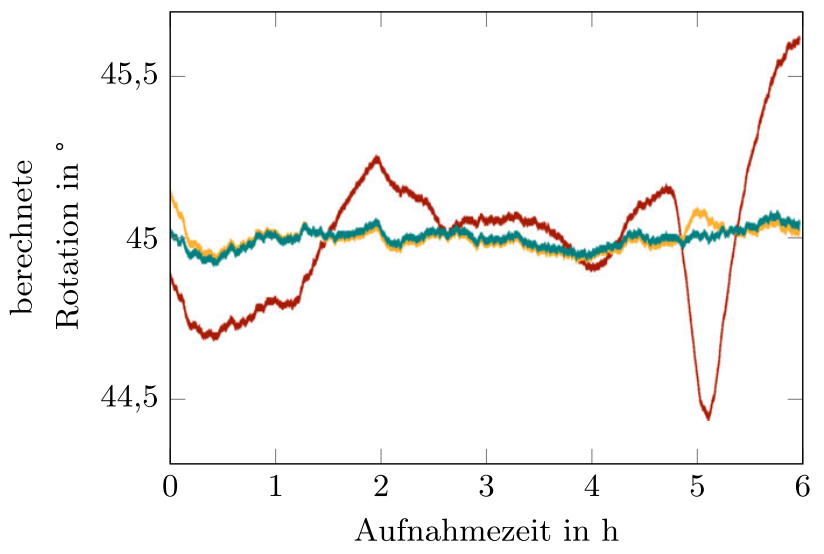

Abb. 5: Langzeitverhalten (6 Stunden) des Ausgangssignals eines einzelnen Pixels unter Verwendung eines Moving-Average-Filters mit einer Filterlänge von $N=1000$ : ohne Korrektur (rot), Korrektur mit Signal der Fotodiode (gelb), zusätzliche Korrektur mit Temperatursensor (grün).

an, weshalb die berechnete Rotation nicht von $45^{\circ}$ abweichen sollte. Das unkorrigierte Signal weist über den sechsstündigen Zeitraum der Messung jedoch Schwankungen von $\pm 0,5^{\circ}$ auf, was einem Achtel des $\pm 4^{\circ}$ umfassenden Messbereichs entspricht. Die Korrektur über die gemessene Lichtleistung der Fotodiode verringert die Schwankungen deutlich auf etwa $\pm 0,1^{\circ}$. Durch die zusätzliche Korrektur der Kameraempfindlichkeit mittels des Temperatursensors in der Kamera können weitere, wenn auch kleinere Verbesserungen erzielt werden.

\subsection{Magnetisierungsverhalten des Faraday-Kristalls}

Der Faraday-Kristall weist bei näherer Betrachtung mehrere unerwartete Eigenschaften auf, die bei einer Messung beachtet werden müssen. Das ferromagnetische Verhalten des Kristalls kann dabei sowohl in makroskopischer als auch mikroskopischer Hinsicht Schwierigkeiten für quantitative Messungen verursachen.

Bei makroskopischer Betrachtung zeigt der Kristall wie in Abbildung 6 A dargestellt im vom Hersteller angegebenen Arbeitsbereich von $\pm 2 \mathrm{kA} \mathrm{m}^{-1}$ einen ausgeprägten Hystereseverlauf für die berechnete Rotation gegenüber einem anregenden Magnetfeld. Ein Hystereseverhalten bedeutet bei bloßer Messung der Rotation aber eine erhöhte Unsicherheit bei der Zuordnung der entsprechenden Feldstärke. Dies ist gleichbedeutend mit einer Vergrößerung des Amplitudenfehlers und somit eine Einschränkung für quantitative Messungen. In der Folge wurde daher der Arbeitsbereich verkleinert, um die Hysterese zu verringern. 

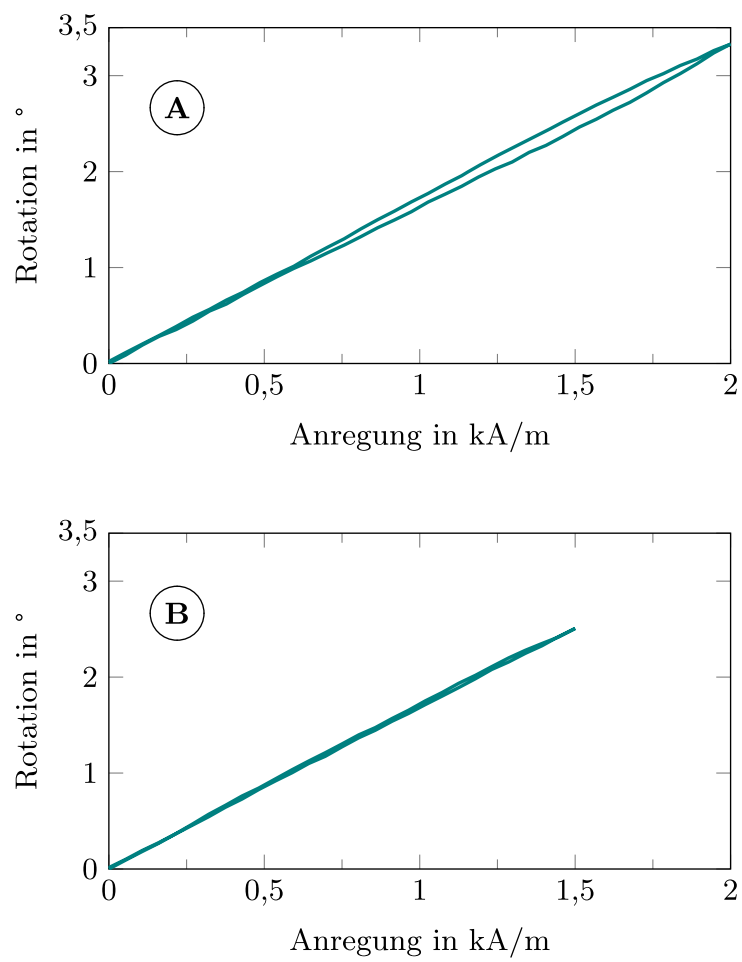

Abb. 6: Rotation in Abhängigkeit der magnetischen Feldstärke: (A) im vom Hersteller angegebenen Arbeitsbereich mit ausgeprägter Hysterese, (B) im reduzierten Arbeitsbereich mit vernachlässigbarer Hysterese.

In Abbildung 6 B ist zu sehen, dass bei einer Reduzierung des Arbeitsbereiches auf $\pm 1,5 \mathrm{kA} \mathrm{m}^{-1}$ die Hysterese als vernachlässigbar angesehen werden kann.

Zusätzlich weist der Kristall auch bei mikroskopischer Betrachtung unerwartetes magnetisches Verhalten auf. Abbildung 7 zeigt die Intensitäts- beziehungsweise Rotationsverteilung eines Ausschnitts der Kristalloberfläche bei einer homogenen Anregung von $0 \mathrm{kA} \mathrm{m}^{-1}$ und $2 \mathrm{kA} \mathrm{m}^{-1}$. Da die inhärente Struktur des Kristalls selbst zur berechneten Rotation beiträgt, kann nicht ohne Weiteres von der Information eines Pixels auf die extern anregende Feldstärke geschlossen werden. Darüber hinaus verschiebt und ändert sich das Muster bei einer Erhöhung der Anregung, wodurch auch ein einfaches Mapping nicht zielführend wäre.

Genauere Aussagen über das Verhalten des Musters können getroffen werden, indem statt der zweidimensionalen Rotationsverteilung dessen Fouriertransformierte betrachtet wird. Hiermit können für eine bestimmte Anregung alle auftretenden räumlichen Frequenzen - ausgedrückt in Linienpaaren pro Millimeter - ermittelt werden. Über die Mittenfrequenz $f_{\mathrm{m}}$ und die maximale Abweichung $\Delta f$ lässt sich die Gesamtheit der auftretenden Frequenzen über den Bereich $B^{\prime}$ mit $B^{\prime}=f_{\mathrm{m}} \pm \Delta f$ beschrei-
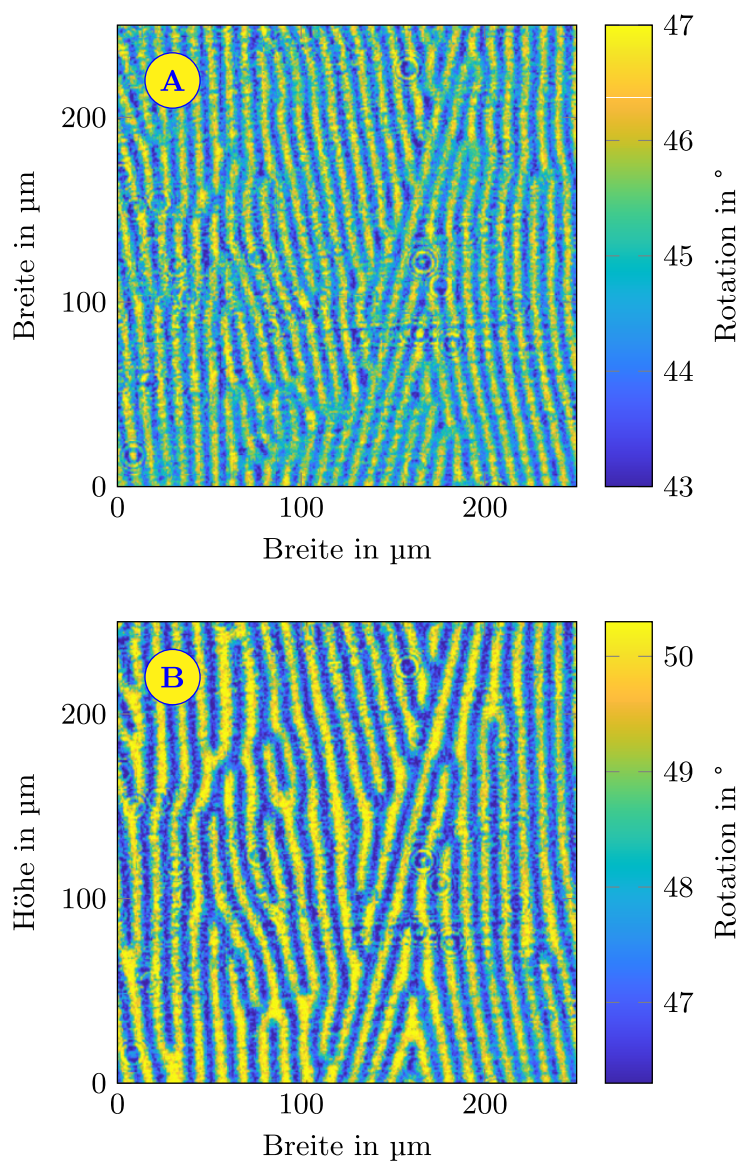

Abb. 7: Inhärente magnetische Domänenstruktur (ferromagnetisches Muster) des Kristalls bei einer Anregung von $0 \mathrm{kA} / \mathrm{m}$ in (A) und $2 \mathrm{kA} / \mathrm{m}$ in (B).

ben. Für die mit einer Anregung von $0 \mathrm{kA} \mathrm{m}^{-1}$ gemessene Struktur in Abbildung 7 A treten lediglich Frequenzen im Bereich $B_{0 \mathrm{kA} \mathrm{m}^{-1}}^{\prime}=112,5 \pm$ 10,5 Linienpaare pro Millimeter auf. Eine Erhöhung der Anregung verursacht neben einer generellen Zunahme der Faraday-Rotation eine Verbreiterung des Bereichs $B^{\prime}$ sowie eine Verschiebung von $f_{\mathrm{m}}$ in Richtung niedrigerer Frequenzen. Im reduzierten Arbeitsbereich von $\pm 1,5 \mathrm{kA} \mathrm{m}^{-1}$ ändert sich das Muster verhältnismäßig wenig. So liegen die Ortsfrequenzen bei einer Anregung von $1,5 \mathrm{kA} \mathrm{m}^{-1}$ im Bereich $B_{1,5 \mathrm{kA} \mathrm{m}^{-1}}^{\prime}=104 \pm 13,5$ Linienpaare pro Millimeter. Bei einer weiteren Erhöhung der Anregung ändert sich das Muster deutlicher. Für eine anregende Feldstärke von $2 \mathrm{kA} \mathrm{m}^{-1}$ enthält die Struktur nach Abbildung 7 (B) beispielsweise die Frequenzen im Bereich $B_{2 \mathrm{kA} \mathrm{m}^{-1}}^{\prime}=91,5 \pm 19,5$ Linienpaare pro Millimeter. Bei einer weiteren Erhöhung der Anregung verliert das Muster seine Regelmäßigkeit vollständig. Innerhalb des reduzierten Arbeitsbereiches kann die Regelmäßigkeit jedoch genutzt werden, indem statt der Information einzelner Pixel der Mittelwert über Cluster benachbarter Pixel ausgewertet 


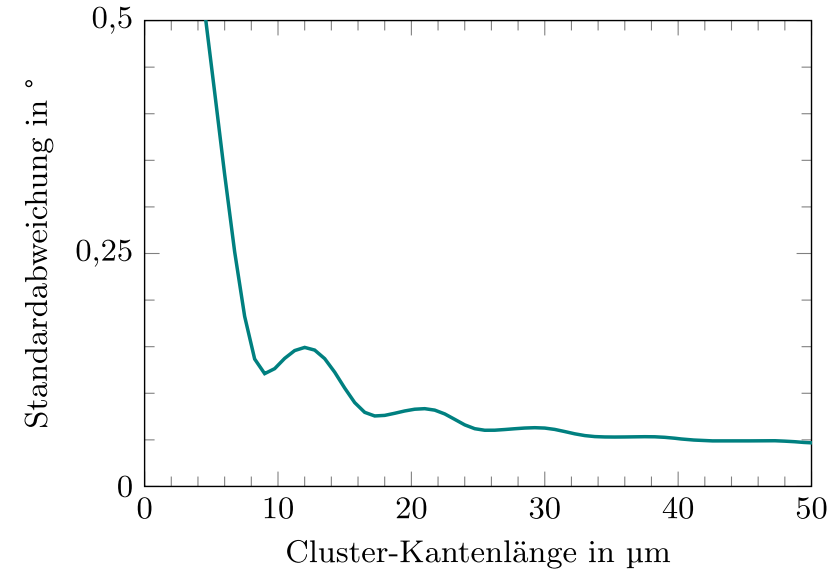

Abb. 8: Mit optischer Vergrößerung ermittelte Standardabweichung in Abhängigkeit der Cluster-Kantenlänge bei einer Anregung von $1,5 \mathrm{kA} / \mathrm{m}$.

wird. Abbildung 8 zeigt für eine Anregung von $1,5 \mathrm{kA} \mathrm{m}^{-1}$ die interpolierte Standardabweichung in erzielten Rotationswinkeln für verschiedene quadratische Cluster in $\mathrm{Ab}$ hängigkeit von deren Kantenlänge. Deren Verlauf verhält sich ähnlich zu einer Sinc-Funktion. Die lokalen Minima entsprechen dabei einer Kantenlänge von ganzzahligen Vielfachen einer Linienpaarbreite. Mit zunehmender Kantenlänge nimmt die Welligkeit des Verlaufs der Standardabweichung und damit der Einfluss des Musters auf den Amplitudenfehler ab. Eine Messung mit größerer Clustergröße ermöglicht also eine erhöhte Amplitudenauflösung, verringert aber die Ortsauflösung. Hierbei muss beachtet werden, dass der genaue Verlauf der Standardabweichung von den auftretenden Frequenzen des Musters und damit von der anregenden Feldstärke abhängt. So klingt die Welligkeit für kleinere Anregungen schneller ab. Bei der Abwägung von Amplituden- und Ortsauflösung bezüglich eines Messproblems sollte vom ungünstigsten möglichen Fall, also der maximal zu erwartenden Anregung durch die Probe ausgegangen werden. Für Abbildung 8 wurde die Anregung von $1,5 \mathrm{kA} \mathrm{m}^{-1}$ gewählt, da dies der oberen Grenze des reduzierten Arbeitsbereich entspricht. Hier ist die Welligkeit etwa ab einer Cluster-Kantenlänge von $40 \mu \mathrm{m}$ vernachlässigbar.

\subsection{Erreichbare Amplitudenauflösung für ein gegebenes Messproblem}

Der Amplitudenfehler des vorgestellten Aufbaus ist zunächst sehr ausgeprägt, kann aber hard- und softwareseitig reduziert werden. Für eine hardwareseitige Verbesserung bietet sich ein Aufbau nach Abbildung 4 an, da hier- bei temperaturbedingte Schwankungen der Intensität der LED korrigiert werden können. Weiterhin kann eine Regelung der LED- und Kamera-Temperaturen beispielsweise durch Peltier-Elemente angewendet werden. Softwareseitig kann die Amplitudenauflösung durch Reduktion des Rauschens und Verringerung der Wirkung der Domänenstruktur verbessert werden. Durch diese Maßnahmen verschlechtert sich allerdings die zeitliche respektive räumliche Auflösung entsprechend. Die Frage nach dem Amplitudenfehler ist demnach abhängig von den Messanforderungen bezüglich räumlicher und zeitlicher Auflösung. Anhand der vorgestellten rechnerischen Zusammenhänge kann diese aber einfach ermittelt werden.

\section{Zusammenfassung}

Ein FRM ist ein Messsystem, das zur zerstörungsfreien Messung magnetischer Mikrostrukturen verwendet werden kann. Es ist potentiell sehr schnell messend, örtlich fein auflösend und eingeschränkt als quantitatives Messsystem einsetzbar. In diesem Beitrag wurde zunächst ein geschwindigkeitsoptimierter Aufbau vorgestellt, der für qualitative Messungen in der Qualitätssicherung reale Anwendung findet. Anschließend wurde ein generalisierter Aufbau präsentiert und charakterisiert, um das Potential für quantitative Messungen zu ermitteln. Dabei stellte sich heraus, dass das Potential eines FRMs als quantitatives Messsystem verwendet zu werden zunächst sehr eingeschränkt und zudem mit einer Verringerung von örtlicher und zeitlicher Auflösung verknüpft ist. Somit hängt die Eignung eines FRMs für quantitative Messungen stark von der Problemstellung ab. Anhand der vorgestellten Ergebnisse kann das Potential eines FRMs abgeschätzt werden. Weiterhin wird gegebenenfalls die Wahl der Komponenten und der Signalverarbeitungsschritte unterstützt. Dabei sollten aber insbesondere der Kristall und die statistischen Eigenschaften seines ferromagnetischen Musters näher untersucht werden.

\section{Literatur}

1. Ripka, P.: Magnetic Sensors and Magnetometers. Artech House Inc., 2001.

2. Hölzl, P. A.; Wiesner, T. ; Zagar, B. G.: Quality assurance for wire connections used in integrated circuits via magnetic imaging. In: 2012 IEEE International Instrumentation and Measurement Technology Conference Proceedings IEEE, 2012, S. 2051-2056.

3. Dietachmayr, F. W.; Hölzl, P. A.; Zagar, B. G. ; Nelhiebel, Michael: Detektion fehlender Bonddrähte in Leistungsbauteilen mittels 
magnetooptischer Bildgebung. In: tm-Technisches Messen 81 (2014), Nr. 10, S. 522-530.

4. Tumanski, S.: Handbook of Magnetic Measurements. Taylor \& Francis Group, LLC, 2011.

5. von Ardenne, M.; Musiol, G. ; Reball, S.: Effekte der Physik und ihre Anwendung. Verlag Harri Deutsch, 1997.

6. Pedrotti, F.; Pedrotti, L.; Bausch, W. ; Schmidt, H.: Introduction to Optics. Pearson Education Company, 1993.

7. Egger, J.; Zagar, B.: In-Line Signal Processing of Faraday Magnetometer Scans. In: Proceedings of EUROCAST 2017, 2017.

8. Matesy GmbH: MO-Sensoren. 2019. - https://matesy.de/de/ produkte/materialien/mo-sensoren-magnetooptik; Abgerufen: 02.09.2019.

9. Northrop Grumman Synoptics: TGG (Terbium Gallium Garnet). 2019. - https://www.northropgrumman.com/ BusinessVentures/SYNOPTICS/Products/SpecialtyCrystals/ Pages/TGG.aspx; Abgerufen: 02.09.2019.

\section{Autoreninformationen}

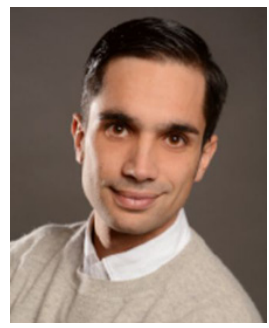

\section{Ruben Piepgras}

Johannes Kepler Universität Linz, Institut für Elektrische Messtechnik, Altenberger Straße 69, 4040 Linz, Austria ruben.piepgras@jku.at

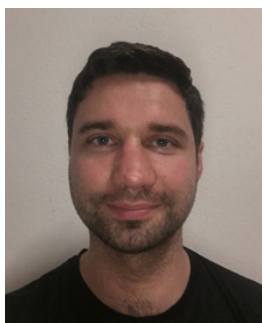

\section{Johannes Egger}

Johannes Kepler Universität Linz, Institut für Elektrische Messtechnik, Altenberger Straße 69, 4040 Linz, Austria office.imt@jku.at

Johannes Egger schloss sein Studium Mechatronik an der Johannes Kepler Universität Linz im Jahr 2018 ab. In seiner Masterarbeit beschäftigte er sich mit magneto-optischen Messsystemen.

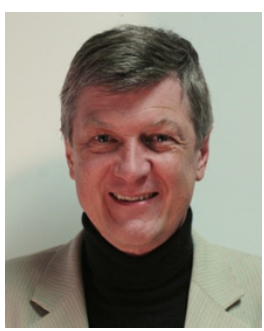

\section{Bernhard G. Zagar}

Johannes Kepler Universität Linz, Institut für Elektrische Messtechnik, Altenberger Straße 69, 4040 Linz, Austria bernhard.zagar@jku.at

Bernhard G. Zagar leitet das Institut für Elektrische Messtechnik an der Johannes Kepler Universität Linz. Seine Interessen sind im Fachgebiet Messtechnik weit gestreut und decken das Thema vom Sensordesign bis hin zur Signalverarbeitung ab. Besondere Schwerpunkte lagen bisher im Bereich der optischen Messtechnik, der Bildverarbeitung und der Magnettomographie.

Ruben Piepgras hat Medizintechnik an der Friedrich-AlexanderUniversität Erlangen-Nürnberg studiert. Seit 2017 ist er Universitätsassistent am Institut für Elektrische Messtechnik der Johannes Kepler Universität Linz. Seine Forschungsinteressen liegen im Bereich der Optik und der magnetischen Messtechnik.

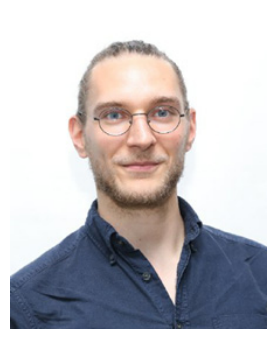

\section{Sebastian Michlmayr}

Johannes Kepler Universität Linz, Institut für Elektrische Messtechnik, Altenberger Straße 69, 4040 Linz, Austria sebastian.michlmayr@gmail.at
Sebastian Michlmayr schloss sein Studium der Mechatronik im Jahr 2018 an der Johannes Kepler Universität Linz ab. Seine Interessensgebiete umfassen optische Messtechnik und Bildverarbeitung. 